\title{
Synchronization Research of Different-structure Chaotic System based on Sliding Mode Variable Structure Control
}

\author{
ZHU Lihong ${ }^{a}{ }^{*}$, KUANG Jiangming ${ }^{b}$ and WEI Jie ${ }^{c}$ \\ The Engineering and Technical College of Chengdu University of Technology, \\ LeShan 614007, SiChuan, P. R. China \\ acczhulihong@163.com, b291806757@qq.com, c64431034 @qq.com
}

Keywords: Sliding mode variable structure; Different-structure chaotic system; Synchronization; The approaching law.

Abstract. In this paper, a nonlinear control method used to make two different chaotic systems achieve synchronization within finite time is designed based on the sliding mode variable structure control theory and the driving response control method. In this method, the reaching law of the sliding mode variable structure may be adjusted to make the sliding mode become stable at a given moment, and make the state error on the sliding mode surface converge to zero globally and rapidly in the form of terminal sliding mode, so as to achieve speedy and accurate synchronization of the different-structure chaotic system. The theoretical analysis and the simulation result verify the availability of the method together.

\section{Introduction}

As a nonlinear control mode ${ }^{[1]}$, in the sliding mode variable structure control, the discontinuous sliding control is used to make state variables of the system slide to the desired points along the specified phase locus. Synchronization of two different chaotic systems must be achieved within finite time. Therefore, synchronous control research of the different-structure chaotic system has important practical significance and appliance value in many fields such as physics ${ }^{[2]}$, communication, information science, medical science, biological and technical science, social sciences ${ }^{[3]}$. Because control parameters of the phase locus in the chaotic system are independent of external disturbance, the different-structure reaching law on the sliding-mode surface makes deviation between two systems converge to zero in a shorter time, and its invariance is superior to robustness.

\section{Synchronization Model}

Suppose the driving system of the chaotic system is ${ }^{[4,5]}$ :

$x=f(x)$

Where $x=[x 1, x 2, \ldots, x n] T \in R n ; f(x)=[f 1(x), f 2(x), \ldots, f n(x)] T \in R n ; x$ and $f(x)$ are the state variable and the smooth nonlinear vector field of Equation (1).

Its response system is combined with the control law, which is expressed as follows:

$\hat{y}=\boldsymbol{g}(\boldsymbol{y})+\boldsymbol{u}$

Where $\mathrm{y}=[\mathrm{y} 1, \mathrm{y} 2, \ldots, \mathrm{yn}] \mathrm{T} \in \mathrm{Rn} ; \mathrm{g}(\mathrm{y})=[\mathrm{g} 1(\mathrm{y}), \mathrm{g} 2(\mathrm{y}), \ldots, \mathrm{gn}(\mathrm{y})] \mathrm{T} \in \mathrm{Rn} ; \mathrm{y}$ and $\mathrm{g}(\mathrm{y})$ are the state variable and the smooth nonlinear vector field of Equation (2). $u=[u 1, u 2, \ldots, u n] T \in R n$ is the controller designed for synchronization of Equation (1) and Equation (2). Suppose the error between Equation (1) and Equation (2) is $\mathrm{e}=\mathrm{y}-\mathrm{x}$, the error vector is $\mathrm{e}=[\mathrm{e} 1, \mathrm{e} 2, \ldots$, en $] \mathrm{T}$, if Equation (1) is subtracted from Equation (2), the state equation of the error system can be derived:

$\Leftrightarrow=g(x+e)-f(x)+u$

Where \& $[\&, \&, \mathrm{~L}, \&]^{T}$.

Synchronous control objective: Two random different-structure chaotic systems are selected, and $\mathrm{f}(\mathrm{x}) \neq \mathrm{g}(\mathrm{y})$ is true in Equation (1) and Equation (2); the applicable control strategy $\mathrm{u}$ is designed to make 
the following expression be true for random original state $\boldsymbol{x}(0)$ and $\boldsymbol{y}(0):\|\boldsymbol{e}(t)\|=\|\boldsymbol{y}(t)-\boldsymbol{x}(t)\|=0\left(t \geq t_{\mathrm{s}}\right)$. That is, the state error is decreased to zero at the accurate moment, so as to make two different-structure chaotic systems achieve precise synchronization ${ }^{[6]}$.

Design of synchronous controller of different-structure chaotic system based on sliding mode variable structure control method

Suppose the switching function is:

$\boldsymbol{s}=\boldsymbol{\alpha}+\boldsymbol{\alpha} \boldsymbol{e}+\boldsymbol{\beta} \boldsymbol{M}(e, q, p)$

Where $\boldsymbol{\alpha}=\operatorname{diag}\left[\alpha_{1}, \alpha_{2}, \ldots, \alpha_{n}\right] ; \boldsymbol{\beta}=\operatorname{diag}\left[\beta_{1}, \beta_{2}, \ldots, \beta_{n}\right]\left(\alpha_{i}, \beta_{i}>0\right) ; \boldsymbol{M}(e, q, p)=\left[e_{1}^{q_{1} / p_{1}}, e_{2}^{q_{2} / p_{2}}, \ldots, e_{n}^{q_{n} / p_{n}}\right]^{T}$, $q_{i}$ and $p_{i}\left(q_{i}<p_{i}\right)$ are positive odd numbers, $i=1,2, \ldots, n$.

When $\mathrm{s}$ is equal to zero, $s \&=0$ is true, derivation of Equation (4) is performed to get the expression $\&=\alpha \hat{\alpha}+\boldsymbol{\beta} \boldsymbol{\alpha} \boldsymbol{\alpha}$, and the following expression can be derived in combination with Equation (3):

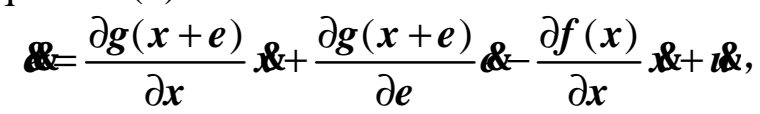

So:

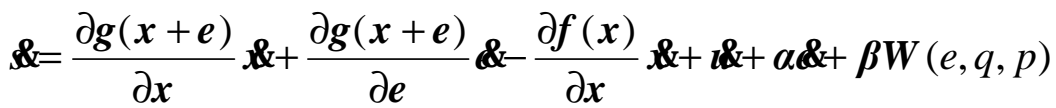

Where $\boldsymbol{W}(e, q, p)=\frac{d \boldsymbol{M}(e, q, p)}{d t}=\boldsymbol{N} \oint$ and $\boldsymbol{N}=\operatorname{diag}\left[\frac{q_{1}}{p_{1}} e_{1}^{q_{1} / p_{1}-1}, \frac{q_{2}}{p_{2}} e_{2}^{q_{2} / p_{2}-1}, \ldots, \frac{q_{n}}{p_{n}} e_{n}^{q_{n} / p_{n}-1}\right]$, Equation

and Equation (3) are substituted into Equation (5), so Equation (6) is derived:

$\delta=\left(\frac{\partial g(x+e)}{\partial x}-\frac{\partial f(x)}{\partial x}\right) f(x)+\left(\frac{\partial g(x+e)}{\partial e}+\alpha+\beta N\right)(g(x+e)-f(x)+u)+\delta$

Suppose the reaching law of the sliding mode variable structure is ${ }^{[7]}$ :

$\&=-\frac{1}{2} \varepsilon \cdot s-\omega L(s, \eta)$

Where $\boldsymbol{s}=\left[s_{1}, s_{2}, \ldots, s_{n}\right]^{T}, \quad \boldsymbol{\varepsilon}=\operatorname{diag}\left[\varepsilon_{1}, \varepsilon_{2}, \ldots, \varepsilon_{\mathrm{n}}\right], \varepsilon_{i}>0 ; \boldsymbol{\omega}=\operatorname{diag}\left[\omega_{1}, \omega_{2}, \ldots, \omega_{n}\right], \omega_{i}>0 ;$

$\boldsymbol{L}(s, \eta)=\left[L_{1}\left(s_{1}, \eta_{1}\right), L_{2}\left(s_{2}, \eta_{2}\right), \ldots, L_{n}\left(s_{n}, \eta_{n}\right)\right]^{T}=\left[2^{-\eta_{1}} s_{1}^{2 \eta_{1}-1}, 2^{-\eta_{2}} s_{2}^{2 \eta_{2}-1}, \ldots, 2^{-\eta_{n}} s_{n}^{2 \eta_{n}-1}\right]^{T}$,

$0<\eta_{i}<1 ; 1 \leq i \leq n$ 。

Theorem In synchronization of two different-structure chaotic systems, the sliding mode variable structure control method is used, and the reaching law of the sliding mode is selected as per Equation (7) so as to make the state error reach the sliding mode surface $s$ within finite time, while the sate error reaching law on the sliding mode surface is selected as Equation (4) may make the error converge to zero within finite time.

Proof: Suppose Lyapunov function is $V_{i}=\frac{1}{2} s_{i}^{2}$, which is derived in combination with Equation (7):

$$
\begin{aligned}
& V_{i}^{\mathcal{\alpha}}=S_{i} \underset{1}{\boldsymbol{\alpha}} \\
& =S_{i}\left(-\frac{1}{2} \varepsilon_{i} s_{i}-\omega_{i} 2^{-\eta_{i}} S_{i}^{2 \eta_{i}-1}\right)=-\frac{1}{2} \varepsilon_{i} s_{i}^{2}-\omega_{i} 2^{-\eta_{i}} S_{i}^{2 \eta_{i}} \\
& =-\frac{1}{2} \varepsilon_{i} s_{i}^{2}-\omega_{i} 2^{-\eta_{i}} s_{i}^{2 \eta_{i}}=-\varepsilon_{i} \frac{1}{2} s_{i}^{2}-\omega_{i}\left(\frac{1}{2} s_{i}^{2}\right)^{\eta_{i}} \\
& =-\varepsilon_{i} V_{i}-\omega_{i} V_{i}^{\eta_{i}}
\end{aligned}
$$


Because of $\varepsilon_{i}>0, \quad \omega_{i}>0, \quad \eta_{i}>0, \quad V_{i}=\frac{1}{2} s_{i}^{2}>0$ and $V_{i}^{\eta_{i}}=\left(\frac{1}{2} s_{i}^{2}\right)^{\eta_{i}}>0, \quad \alpha_{i}^{\alpha}=-\varepsilon_{i} V_{i}-\omega_{i} V_{i}^{\eta_{i}}<0$ can be derived, it is can be seen from the above expression that the sliding mode surface $s_{i}$ is stable at the reaching law in Equation (7).

On the basis of Equation (8), $\dot{V}_{i}=-\varepsilon_{i} V_{i}-\omega_{i} V_{i}^{\eta_{i}}$ can be derived, namely $V_{i}+\varepsilon_{i} V_{i}+\omega_{i} V_{i}^{\eta_{i}}=0$, and it can be transformed as follows:

$$
V_{i}^{-\eta_{i}} \frac{d V_{i}}{d t}+\varepsilon_{i} V_{i}^{1-\eta_{i}}=-\omega_{i}
$$

Suppose $R_{i}=V_{i}^{1-\eta_{i}}, \frac{d R_{i}}{d t}=\left(1-\eta_{i}\right) V_{i}^{-\eta_{i}} \frac{d V_{i}}{d t}$ can be derived, and it is substituted into Equation (9) and simplified:

$$
\frac{d R_{i}}{d t}+\left(1-\eta_{i}\right) \varepsilon_{i} R_{i}=-\left(1-\eta_{i}\right) \omega_{i}
$$

The first-order differential equation is solved to get the following:

$$
R_{i}(t)=-\frac{\omega_{i}}{\varepsilon_{i}}+\frac{\omega_{i}}{\varepsilon_{i}} e^{-\left(1-\eta_{i}\right) \varepsilon_{i} t}+R_{i}(0) e^{-\left(1-\eta_{i}\right) \varepsilon_{i} t}
$$

When $s_{\mathrm{i}}=0, V_{i}=0$ and $R_{i}=0$ are true, the moment $t=t_{\mathrm{si}}$ is supposed, the following can be derived based on Equation (3) to Equation (11):

$$
t_{s i}=\frac{1}{\left(1-\eta_{i}\right)} \ln \frac{\omega_{i}+\varepsilon_{i} V_{i}(0)}{\omega_{i}}
$$

Where $V_{\mathrm{i}}(0)$ is the original state of Equation (8). It can be known from Equation (12) that, at the moment $t_{\mathrm{si}}\left(s_{\mathrm{i}}=0\right)$, the adjustable parameters $\eta_{i}, \omega_{i}$ and $\varepsilon_{i}$ are used to determine the moment $s_{\mathrm{i}}$ when the sliding mode surface is reached; when $s_{\mathrm{i}}=0$ is true, the expression $s_{i}=\dot{e_{i}}+\alpha_{i} e_{i}+\beta_{i} e_{i}^{q_{i} / p_{i}}=0$ can be derived from Equation (4); on the sliding mode surface of the terminal, the adjustable parameters $\alpha_{i}, \beta_{i}$, $q_{i}$ and $p_{i}$ can be used to determine the time in which the state error converges to zero, and the differential equation is solved to get the moment when $e_{\mathrm{i}}$ converges to zero:

$$
t_{e i}=\frac{p_{i}}{\alpha_{i}\left(p_{i}-q_{i}\right)} \ln \frac{\beta_{i}+\alpha_{i} e_{i}^{\left(p_{i}-q_{i}\right) / p_{i}}(0)}{\beta_{i}}
$$

The control law $\boldsymbol{u}$ can be derived based on Equation (6) and Equation (7):

$$
\begin{aligned}
& \boldsymbol{u} \leqslant=-\left(\frac{\partial g(x+e)}{\partial x}-\frac{\partial f(x)}{\partial x}\right) f(x)-\left(\frac{\partial g(x+e)}{\partial e}+\alpha+\beta N\right)(g(x+e) \\
& -f(x)+u)-\frac{1}{2} \varepsilon \cdot s-\omega L(s, \eta)
\end{aligned}
$$

In addition, the filter of the control law $\boldsymbol{u}$ can be obtained from Equation (14):

$$
\begin{aligned}
& \boldsymbol{L} \&+B(x, e) u=-A(x, e) f(x)-B(x, e)(g(x+e)-f(x))-\frac{1}{2} \varepsilon \cdot s-\omega L(s, \eta) \\
& A(x, e)=\frac{\partial g(x+e)}{\partial x}-\frac{\partial f(x)}{\partial x}, \quad B(x, e)=\frac{\partial g(x+e)}{\partial e}+\alpha+\beta N
\end{aligned}
$$

\section{Simulation analysis}

In this section, Chen system and Liu system as examples are studied for the synchronization problem of two different-structure chaotic systems, and then the above theory are proven ${ }^{[8,9]}$.

Suppose Chen system is the driving system: 


$$
\left\{\begin{array}{l}
\hat{\alpha}=\rho\left(x_{2}-x_{1}\right) \\
\dot{\alpha}=(\mu-\rho) x_{1}-x_{1} x_{3}+\mu x_{2} \\
\dot{\alpha}=x_{1} x_{2}-\delta x_{3}
\end{array}\right.
$$

When $\rho=35, \delta=3$ and $\mu=28$, Chen system lies in the chaotic state. The phase diagram at the chaotic state is illustrated as follows:

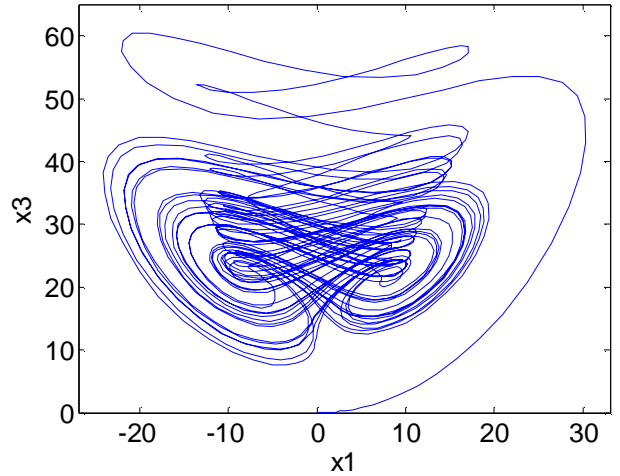

Fig. 1 Phase Diagram of Chen System's Variable $x_{11}$

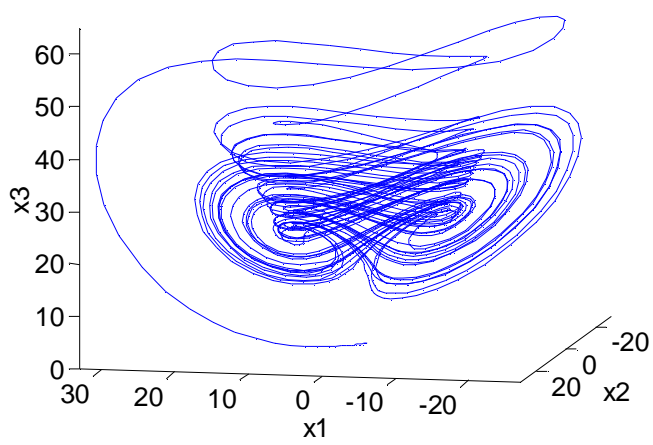

Fig. 2 Phase Diagram of Chen System's Variables $x_{1} y_{1} z_{1}$

Suppose Liu system is the response system, the following expression is derived:

$$
\left\{\begin{array}{l}
\propto=a\left(y_{2}-y_{1}\right)+u_{1} \\
\psi_{2}=b y_{1}-y_{1} y_{3}+u_{2} \\
\&=y_{1}^{2}-c y_{3}+u_{3}
\end{array}\right.
$$

In Liu system, the system lies in the chaotic state in the case of $a=10, b=40$ and $c=2.5$. The phase diagram at the chaotic state is illustrated in Fig.3 and Fig.4:

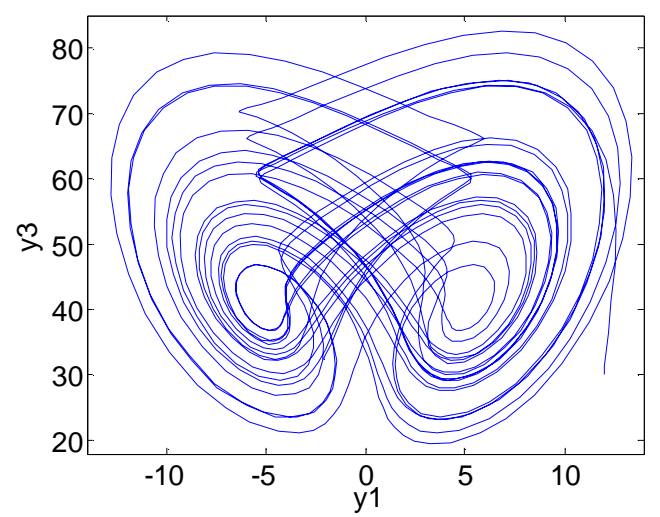

Fig. 3 Phase Diagram of Chen System's Variables $x_{2} z_{2}$

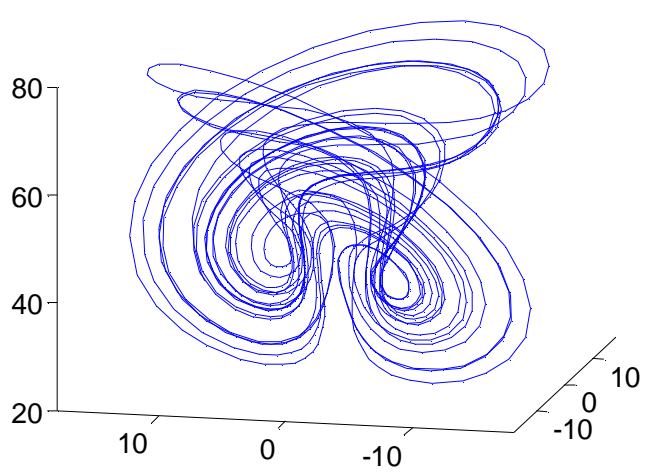

Fig. 4 Phase Diagram of Chen System's Variables $x_{2} y_{2} z_{2}$

In the response system, $\boldsymbol{u}=\left[u_{1}, u_{2}, u_{3}\right]^{T}$ is the control law of the sliding mode variable structure selected, the following can be derived from Equation (17) and Equation (18):

$$
\boldsymbol{f}(\boldsymbol{x})=\left[\begin{array}{l}
\rho\left(x_{2}-x_{1}\right) \\
(\mu-\rho) x_{1}-x_{1} x_{3}+\mu x_{2} \\
x_{1} x_{2}-\delta x_{3}
\end{array}\right], \quad \boldsymbol{g}(\boldsymbol{x}+\boldsymbol{e})=\left[\begin{array}{l}
a\left(\left(x_{2}+e_{2}\right)-\left(x_{1}+e_{1}\right)\right) \\
b\left(x_{1}+e_{1}\right)-\left(x_{1}+e_{1}\right)\left(x_{3}+e_{3}\right) \\
\left(x_{1}+e_{1}\right)^{2}-c\left(x_{3}+e_{3}\right)
\end{array}\right]
$$

Namely $\frac{\partial \boldsymbol{f}(\boldsymbol{x})}{\partial \boldsymbol{x}}=\left[\begin{array}{ccc}-\rho & \rho & 0 \\ \mu-\rho-x_{3} & \mu & -x_{1} \\ x_{2} & x_{1} & -\delta\end{array}\right], \frac{\partial \boldsymbol{g}(\boldsymbol{x}+\boldsymbol{e})}{\partial \boldsymbol{x}}=\frac{\partial \boldsymbol{g}(\boldsymbol{x}+\boldsymbol{e})}{\partial \boldsymbol{e}}=\left[\begin{array}{ccc}-a & a & 0 \\ b-\left(x_{3}+e_{3}\right) & 0 & -\left(x_{1}+e_{1}\right) \\ 2\left(x_{1}+e_{1}\right) & 0 & -c\end{array}\right]$,

According to $A(x, e)=\frac{\partial \boldsymbol{g}(\boldsymbol{x}+\boldsymbol{e})}{\partial \boldsymbol{x}}-\frac{\partial \boldsymbol{f}(\boldsymbol{x})}{\partial \boldsymbol{x}}$ in Equation (16), $\boldsymbol{B}(\boldsymbol{x}, \boldsymbol{e})=\frac{\partial \boldsymbol{g}(\boldsymbol{x}+\boldsymbol{e})}{\partial \boldsymbol{e}}+\boldsymbol{\alpha}+\boldsymbol{\beta N}$ can be derived: 


$$
\begin{aligned}
\boldsymbol{A}(\boldsymbol{x}, \boldsymbol{e}) & =\left[\begin{array}{ccc}
-a+\rho & a-\rho & 0 \\
b-\mu+\rho-e_{3} & -\mu & -e_{1} \\
2\left(x_{1}+e_{1}\right) & -x_{1} & \delta-c
\end{array}\right] \\
\boldsymbol{B}(\boldsymbol{x}, \boldsymbol{e}) & =\left[\begin{array}{ccc}
\alpha_{1}-a+\beta_{1} \frac{q_{1}}{p_{1}} e_{1}^{q_{1} / p_{1}-1} & a & 0 \\
b-\left(x_{3}+e_{3}\right) & \alpha_{2}+\beta_{2} \frac{q_{2}}{p_{2}} e_{2}^{q_{2} / p_{2}-1} & -\left(x_{1}+e_{1}\right) \\
2\left(x_{1}+e_{1}\right) & 0 & \alpha_{3}-c+\beta_{3} \frac{q_{3}}{p_{3}} e_{3}^{q_{3} / p_{3}-1}
\end{array}\right]
\end{aligned}
$$

$\boldsymbol{f}(\boldsymbol{x})$ and $\boldsymbol{g}(\boldsymbol{x}+\boldsymbol{e})$ in Equation (19), $\boldsymbol{A}(\boldsymbol{x}, \boldsymbol{e})$ in Equation (20), B(x,e) in Equation (21) and $\boldsymbol{s}$ in Equation (7) are substituted into the control law equation (5), and in Equation (4), $\left[\alpha_{1}, \alpha_{2}, \alpha_{3}\right]=[4,3,3],\left[\beta_{1}, \beta_{2}\right.$, $\left.\beta_{3}\right]=[20,330 / 7,42],\left[q_{1}, q_{2}, q_{3}\right]=[3,7,5],\left[p_{1}, p_{2}, p_{3}\right]=[5,11,7]$; in Equation $(7),\left[\varepsilon_{1}, \varepsilon_{2}, \varepsilon_{3}\right]=[20,10,15]$, $\left[\omega_{1}, \omega_{2}, \omega_{n}\right]=[6,3,7],\left[\eta_{1}, \eta_{2}, \eta_{3}\right]=[7 / 11,3 / 5,5 / 7]$. The original values of Equation (17) and Equation $(18)$ are $\left[x_{1}(0), x_{2}(0), x_{3}(0)\right]=[0.01,0.02,0.01]$ and $\left[y_{1}(0), y_{2}(0), y_{3}(0)\right]=[12,13,30]$, respectively. The above parameters are substituted into Equation (4) to get $s(0)$ and the time $t_{\mathrm{e} i}$; and $s(0)$ is substituted into $V_{\mathrm{i}}=0.5 \mathrm{~s}_{\mathrm{i}}{ }^{2}$, to get $V_{\mathrm{i}}(0) ; V_{\mathrm{i}}(0)$ and parameters $\boldsymbol{\omega}, \boldsymbol{\varepsilon}$ and $\boldsymbol{\eta}$ are substituted into Equation (12) to get $\boldsymbol{t}_{\mathrm{si}}$.

Simulation result: the convergence curves of synchronization state errors $e_{1}, e_{2}$ and $e_{3}$ in two chaotic systems are shown in Fig. 5; it can be seen from Fig. 5 that, the moment when three states achieve synchronization is equal to the theoretical calculation result; Fig. 6 shows the relationship curve of the nonlinear control law $u_{1}, u_{2}$ and $u_{3}$ varying with time; Fig. 7 is the synchronization phase diagrams of Liu system and Chen system, and the state variables are $x_{1}, x_{3}$ and $y_{1}, y_{3}$. It can be known from the figure that Liu system tends to be synchronous with Chen system, therefore, the control strategy proposed in this paper can achieve synchronization of two different-structure chaotic systems.
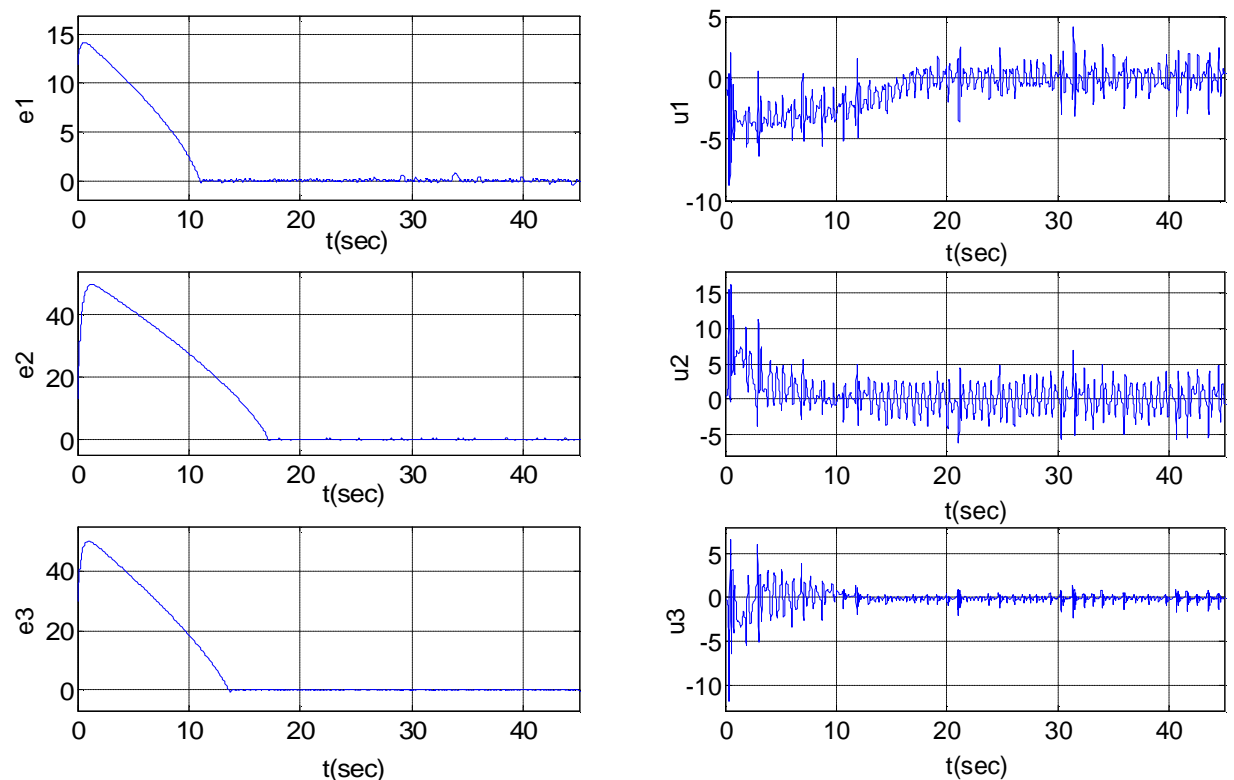

Fig. 5 State Error Convergence Curves of Two Chaotic Systems Fig. 6 Relationship Curve of Control Law $u$ in term of Time 


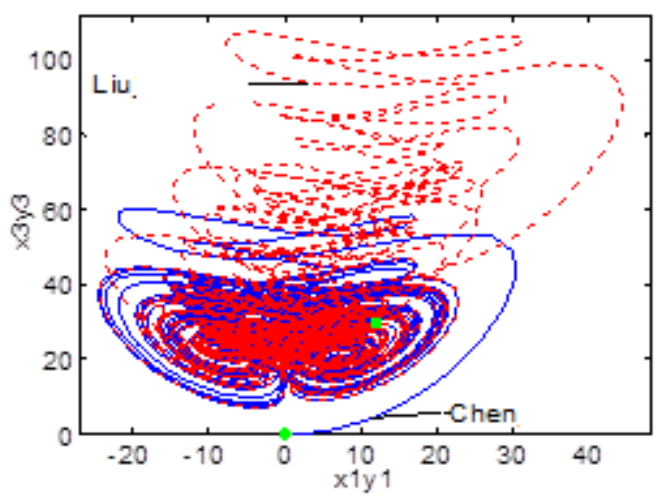

Fig. 7 Synchronization Phase Diagram of Two Systems

\section{Summary}

Experimental result shows that, on the basis of the sliding mode variable structure control method, the nonlinear control strategy proposed in this section is used to achieve synchronization of two different-structure chaotic systems effectively. The time when the sliding mode surface is reached is determined by adjusting parameters $\omega_{\mathrm{i}}, \varepsilon_{\mathrm{i}}$ and $\eta_{\mathrm{i}}$. the time when the state error converges to zero is determined adjusting parameters $\alpha_{\mathrm{i}}, \beta_{\mathrm{i}}, q_{\mathrm{i}}$ and $p_{\mathrm{i}}$. This method has rapid dynamic response and strong robustness, insensitive to parameters and external disturbance.

\section{References}

[1] HU Junda. HU Hui. Study on the Application of Speed Control to Asynchronous Motor Based-on Sliding Mode Variable Structure Control. Large Electric Machine and Hydraulic Turbine.2006,(5):32-35.

[2] Pecora L M, Carroll T L. Synchronization in chaotic Circuits. Physic Review Letters, 1990, 64,(8): 821-824.

[3] WANG Xiaoyan, QU Shaocheng. Synchronization of different structure chaotic systems and its application in secure communication.Application research of computers 2009,26(5):1874-1876.

[4] QI Dong-lian, ZHAO Guang-zhou. Cont rol uncertain continuous-time chaotic dynamical system. Journal of Zhejiang University SCIENCE, 2003, 4(4): 437-440.

[5] Kocarev L, Parlitz U. General approach for chaotic synchron-ixaiton with application to communication. Physics Review letters, 1995, 74(25): 5028-5031.

[6]Hunt B. R., Ott E. and Yorke J.A., Differential generalized synchronization of chaos, Phys.Rev.E, 1997, 55(4):4029-4034.

[7]LIU Jinkun.sliding mode control design and matlab simulation].TSingh university press.2005.

[8]LEI Yan. The simulation reality of chaotic system. Digital technology \& application.2009,(10):105-108.

[9] Yassen M T. Chaos synchronization between two different chaotic systems using active control . Solutions and Fractals, 2005,23(4):131-140. 